\title{
Lateral
}

JOURNAL OFTHE CULTURALSTUDIESASSOCIATION

Michael Mario Albrecht, "Review of 'Make America Meme Again: The Rhetoric of the Alt-Right' by Heather Suzanne Woods \& Leslie A. Hahner (Peter Lang)," Lateral 8.2(2019).

https://doi.org/10.25158/L8.2.18

This content is licensed under a Creative Commons Attribution 4.0 International License. Copyright is retained by authors.

\section{Review of Make America Meme Again: The Rhetoric of the Alt-Right by Heather Suzanne Woods \& Leslie A. Hahner (Peter Lang)}

\section{Michael Mario Albrecht}

\begin{abstract}
In Make America Meme Again, Heather Suzanne Woods and Leslie Hahner highlight the central role that memes play in the contemporary political landscape. Specifically, the authors show the ways in which members of the Alt-right have deftly used memes to forward their political agenda, to recruit new members, and to move the so-called "Overton window" rightward to expand the acceptable field of political discourse. In their schema, memes are not simply distractions from important political issues; rather, they function rhetorically and work to constitute the political field. The authors trace the origins of the Alt-right to the murky depths of the internet and show how discourses that emerged from these shadowy depths were able to appeal to a wider audience and cohere around mainstream political discourse. The authors carefully outline the ways that memes circulate, the rhetorical strategies that the Alt-right uses to deploy them, and the ways they work to disavow any charges of racism or extremism that critics might level against them. For Woods and Hahner, memes are the dominant mechanism through which the Alt-right is able to secure and enact its discursive power. Aware of the bleakness of this particular historical moment, Make America Meme Again provides a cogent argument for the ways the Alt-right has been able harness memetic power while also offering a blueprint through which future scholars and activists might reconfigure the present conjuncture so that the Alt-right does not have a stranglehold over the discursive power of memes in the future.
\end{abstract}

Make America Meme Again: The Rhetoric of the Alt-Right. By Heather Suzanne Woods \& Leslie A. Hahner. New York: Peter Lang Publishing, 2019, pp. 258

(hardcover). ISBN 978-1-4331-5974-9. US List \$89.95.

In the era of Donald J. Trump, a great deal of (mostly virtual) ink has been spilled insisting that tweets, memes, and other defining characteristics of the social media age are mere distractions from truly important issues. Many scholars and pundits bemoan the current state of social media culture and insist that those who wish to defeat Trump and his politics need to cut through the distractions and get to the underlying truth by returning to traditional journalistic practices that existed in the era of print media. In Make America Meme Again, rhetorical scholars Heather Suzanne Woods and Leslie A. Hahner put to rest any claims that memes are simply distractions from the "real" politics and issues to which scholars, journalists, politicians, and pundits need to return.

For Woods and Hahner, memes are precisely the terrain upon which members of the Altright have waged political and cultural war and through which the Alt-right continues to mount political and cultural battles, recruit new members, and bolster the presidency of Donald J. Trump as a symbol of their white nationalist political agenda. The authors argue that "in a number of boards on 4chan and reddit, visual, static memes became a crucial site for advancing not simply the election of Trump but engendering a significant shift in public culture. The Alt-right made tactical use of memes to create a public presence and attract new members" (3). In their framework, memes are not mere distractions; rather, they function rhetorically in ways that constitute publics and effect political ends. Woods and 
Hahner do not equivocate on this point; they insist that "memes are not simply one tactic for the Alt-right-they are the primary rhetorical mechanism grounding its broader work and linking outsiders to its radical views" (5). This statement should be arresting for many scholars of politics and media. There is a wealth of supposedly commonsense consensus among political and media scholars and pundits that the most critical problems in the field are the oligopolistic nature of media ownership and the disinformation campaigns of Fox News or AM talk radio. For many of these scholars, journalists, and pundits, studying memes is a trivial endeavor that should be of secondary importance to these real political economic issues. Make America Meme Again offers a shot across the bow at these myopic understandings of media and politics. Woods and Hahner are not simply trying to add their savvy contribution to media studies, political science, and political communication; they are challenging these fields to rethink many of the very premises that currently undergird them.

Woods and Hahner begin their book by exploring the origins of online meme culture on 4chan and reddit. They maintain that because the sites offer degrees of anonymity or pseudonymity, users are able to transgress boundaries of acceptable behavior and social norms, consequently moving the political "Overton window." They maintain that "both reddit and 4chan thrive off of the anonymity/pseudonymity requisite of the sites, serve simultaneously as producers, hosts, and disseminators of memetic content, and constantly negotiate an ambivalent, antagonistic relationship with the public sphere" (26). Reddit and 4chan were able to thrive as sites where reprehensible content circulated with very little outside supervision; simultaneously these sites constructed ties to the mainstream through cat memes and other seemingly innocuous content. They persuasively demonstrate that while the boards on these sites were bringing Caturdays and Lolcats to the mainstream, they were simultaneously developing a culture of unmitigated free speech that luxuriated in racist, misogynist, homophobic language and imagery.

One of the rhetorically shrewdest characteristics of the Alt-right is its ability to dwell in the ambiguous space between irony and sincerity. Because its members' arguments are often couched in the form of memes, they are often able to claim that if they are posting something outrageous, they are just "doing it for the lulz," and that those who are offended are being too politically correct. Woods and Hahner argue that "for the Alt-right, the deployment of ironic images through lulz supplies rhetorical cover for hate-filled messages" (105). They go on to suggest that "such disavowal recognizes the racism contained in far-right memes but refuses to accept responsibility for conveying racism" (105). As such, the form of the meme provides an alibi for the racist (or sexist or homophobic) content of the meme. The original poster of the meme (or "shitposter" in the Internet patois) can always claim that the meme is just a joke and that it shouldn't be taken seriously. Hahner and Woods quickly dismiss this line of argument; they aver that "white supremacy may be clothed in irony but that dressing does not change its impacts. Ultimately, lulz enables the proliferation of Alt-right discourse and reifies the attitudes of white supremacy" (109). While members of the Alt-right might try to use the polysemic nature of irony to coyly avoid the label "racist," Hahner and Woods reject this gambit and insist that regardless of intent, the racist impacts remain the same.

By the final chapter, Woods and Hahner have meticulously shown the origins of memetic culture, the ways that memes allow the Alt-right to use iconic imagery to their advantage, the rhetorical strategies and tactics the Alt-right employs, memes' modes of circulations, and the ways the Alt-right uses memes to silence the opposition. Over the course of the book, the authors present overwhelming evidence for the entrenched power of the Altright and its decisive advantage by 2019 in the Great Meme Wars. In the conclusion, "The 
Coming Meme Battles," Woods and Hahner note that scholars and activists on the Left are currently working from a huge disadvantage. For them, "the Alt-right is playing a different game than 'normies.' They are winning the meme war, bigly" (217).

The shimmering hope in this otherwise extraordinarily bleak assessment of the present state of contemporary media and politics is the ambivalent nature of memes-they have no fixed valence. While the Right has so far been better at taking up memes, there is nothing inherently conservative about memes. Woods and Hahner point out the seemingly contradictory ability of memes to concomitantly work to unite and divide. They argue that "memes are thus useful in effecting change in two powerful ways: by drawing people together into collectives and by dividing them through chaos, confusion, and antagonism" (212). As such, "memes are an agile, deft and perhaps even dangerous form of communication" (212). However, Woods and Hahner are careful to dispel any facile understanding of memes' lack of fixed valence. They clearly articulate that "memes are not politically neutral. Rather, they are constituted by and through the encultured media ecologies from which they are born and are circulated. In the present tense, those media ecologies skew conservative. That is not to suggest that memes cannot be radical [...] but the present conjuncture of discourse across networks and the rhetorical potency of Altright tactics belies the radicality of the form" (221).

In other words, if scholars, pundits, and political activists want to have any chance the of winning upcoming meme battles, they need to take memes seriously, understand the ways in which they circulate, analyze the rhetorical strategies they employ, and offer strategies of resistance that comport with current media ecologies. The struggle to make memes radical again requires a great deal of hard work on the part of scholars and activists to change the conjuncture to one in which the memetic form does not skew dramatically to the right. Woods and Hahner provide a blueprint for how the Alt-right has been able to transform memes into the dominant tool for their political agenda; subsequent scholars and activists can use this blueprint to work to change the current conjuncture to one more favorable to Leftist politics.
$\underline{\dot{B} \text { Bio }}$
Twitter
Michael Mario Albrecht
Michael Mario Albrecht is a Visiting Instructor of English at the University of South Florida with a PhD from the University of lowa in Communication Studies with a focus in Media Studies. He has held teaching positions at Eckerd College, The University of New Hampshire, and the University of Wisconsin-Milwaukee. His research interests include television studies, masculinity, critical theory, popular culture, and contemporary politics. Masculinity in Contemporary Quality Television (2014) is currently available from Routledge Press.

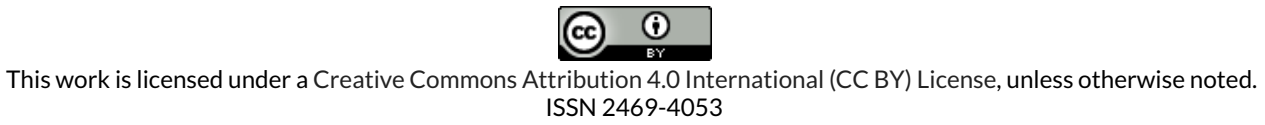

